QUIPURAMAYOC Revista de la Facultad de Ciencias Contables

Vol. 24 N.o 46 pp. 191-199 (2016) UNMSM, Lima - Perú

ISSN: 1560-9103 (versión impresa) / ISSN: 1609-8196 (versión electrónica)

\title{
MODELO ECONOMÉTRICO PARA LA VALORACIÓN ECONÓMICA DEL ECOTURÍSMO: PARQUE NACIONAL DE TINGO MARÍA
}

\author{
ECONOMETRIC MODEL FOR ECONOMIC VALUETION OF ECOTOURISM: \\ TINGO MARÍA NATIONAL PARK \\ Luis Moraies y Chocano* \\ Profesor principal de la Facultad de Ciencias Económicas y Administrativas \\ Aiex Rengifo Rojas** \\ Profesor Auxiliar de la Facultad de Ciencias Económicas y Administrativas \\ DanielGuZm án Rojas ${ }^{* * *}$ \\ Profesor principal de la Facultad de Ciencias Económicas y Administrativas \\ Universidad Nacional Agraria de la Selva - Perú \\ [Recepción: Agosto 2016/ Conformidad: Setiembre 2016]
}

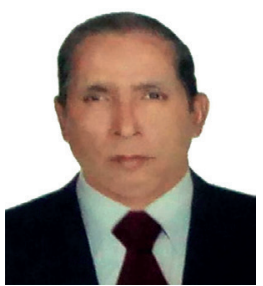

\section{RESUMEN}

La investigación fue realizada, en el Parque Nacional Tingo María y tuvo como objetivo identificar los factores determinantes del valor económico del ecoturismo, generado por la "Cueva de las Lechuzas". Para comprobar la hipótesis de que los aspectos socioeconómicos y la elección de los servicios ambientales de los visitantes, influyen en el valor económico del ecoturismo, se procedió a la aplicación de una encuesta, con cuyos resultados se estimó el modelo micro econométrico de elección binaria, para determinar la disponibilidad a pagar por concepto de ingreso al atractivo turístico, llegándose a establecer que tanto los aspectos socioeconómicos, como los servicios ambientales influyen en la disponibilidad a pagar. Con relación a las características de la elección de los servicios ambientales, se encontró que el principal motivo de la visita a este importante recurso, corresponde a la invitación de un amigo y/o familiar que antes ya visitó la zona. Respecto, al destino de la visita, la mayoría de los encuestados coinciden que la visita a la Cueva de las Lechuzas no constituye el único destino. Por último, el grueso de los visitantes lo clasifica como turismo recreativo y la disponibilidad a pagar es de tres soles por visitante nacional y cinco soles por extranjero.

\section{Palabras Clave:}

Socioeconómico, servicio ambiental, biodiversidad, ecosistema y disponibilidad a pagar.

\begin{abstract}
The research was carried out in the Tingo María National Park and aimed to identify the determinants of the economic value of ecotourism, generated by "Cueva de las Lechuzas". In order to verify the hypothesis that the socioeconomic aspects and the choice of the environmental services of visitors influence the economic value of ecotourism, a survey was carried out. A binary-choice microeconometric model was estimated, to determine the convenience of entry payment to the tourist attraction, achieving to establish that both socioeconomic aspects and environmental services influence the intention of payment. Regarding the characteristics of environmental services choice, it was found that the main reason to visit to this important resource, corresponds to the invitation of a friend and / or family member who previously visited the area. Regarding the destination of the visit, most respondents agree that the Cueva de las Lechuzas visit is not the only destination. Finally, the majority of visitors classifies it as recreational tourism, and the disposition to pay is three soles per national visitor and five soles per foreigner.
\end{abstract}

\section{Keywords:}

Socioeconomic, environmental service, biodiversity, ecosystem and disposition to pay.

\footnotetext{
* Magister en economía - UNMSM. Economista. Email: luismoraleschocano@gmail.com

** Economista. Email: alrero2002@gmail.com

*** Maestro en Ciencias Económicas. Economista. Email: danguzrotm@gmail.com
} 


\section{INTRODUCCIÓN}

El Perú no es ajeno a la problemática ambiental que enfrentan los demás países, por lo que la relación hombre -medio ambiente siempre ha estado vinculada por las actividades de extracción, producción, distribución y consumo de bienes y servicios. Estas actividades desarrolladas por el ser humano contribuyen al mayor acceso tecnológico y por tanto, generan cambios en el bienestar de la población.

Los cambios sustanciales negativos en el bienestar de la población, han empezado a reflejarse a partir de los hechos ocurridos durante la revolución industrial. Desde entonces, el hombre se preocupó por las alteraciones del medio ambiente, generadas por nosotros mismos. El desequilibrio del ecosistema es el principal problema que enfrenta hoy en día la sociedad; ya que, los recursos naturales empiezan a ser cada vez más escasos; llegando al punto de amenazar la existencia misma de los recursos hídricos, forestal, biológicos y otros. La conservación y preservación de hoy en día de esta biodiversidad es de suma importancia, con el fin de asegurar hoy y mañana la provisión de recursos para las futuras generaciones.

La Cueva de las Lechuzas es de uso turístico, recreativo e investigación, como consecuencia de la degradación del ecosistema ha generado una merma en la cantidad y calidad del agua; así como ha ido modificando sustancialmente la belleza paisajística, consecuentemente esto ha conllevando a una disminución en los ingresos económicos de la comunidad beneficiaria.

El objetivo de la investigación, consiste en analizar la influencia de los aspectos socioeconómicos y las características de la elección de los servicios ambientales, sobre el valor económico del ecoturismo de la Cueva de las Lechuzas, por lo que nos ha permitido obtener la disponibilidad a pagar (DAP) por parte los turistas, con el fin de cuidar que no se siga degradando el ecosistema existente en el lugar, por la sobre explotación de los recursos naturales.

\section{MARCO TEÓRICO}

Según Uribe B., Mendieta J. \& otros (2003), el modelo de valoración contingente es en esencia, probabilístico. La probabilidad de una respuesta positiva a una pregunta sobre disponibilidad de pago, depende tanto de: los atributos socioeconómicos del encuestado, edad, ingreso, género, educación, preferencias, etc.-, como de los atributos de calidad $y$ de cantidad del bien ambiental que se ofrece.

El método de valoración contingente supone que el individuo experimenta un mayor nivel de utilidad, si accede a los beneficios que le provee el bien ambiental ofrecido. Si por ejemplo, se piensa en el mejoramiento del ecosistema de la cueva de las lechuzas, por tanto la calidad del aire de la ciudad de Tingo María, mejoraría notablemente, se estima que un individuo tendrá un mayor nivel de bienestar después de la mejora. Esto se expresa como:

$$
\begin{aligned}
& \mu_{i 0}=X_{i 0} \beta+\varepsilon_{i 0} \\
& \mu_{i 1}=X_{i 1} \beta+\varepsilon_{i 1}
\end{aligned}
$$

Donde:

$\mu_{i 0}=$ Es la utilidad del individuo "i” por elegir la opción cero (no visitar la cueva de las lechuzas).

$\mu_{i 1}=$ Es la utilidad del individuo " $i$ " por elegir la opción uno (visitar la cueva de las lechuzas).

$X_{i 0}=$ El vector de variables $\mathrm{Xs}$ relacionados a la opción cero (no visitar la cueva de las lechuzas).

$X_{i 1}=$ El vector de variables $\mathrm{Xs}$ relacionados a la opción uno (visitar la cueva de las lechuzas).

$\varepsilon_{i 0} \wedge \varepsilon_{i 1} \sim N\left(0, \sigma^{2}\right)$, son los términos de error y se distribuyen normalmente con media cero $\left(E\left(\varepsilon_{i 0}\right)=E\left(\varepsilon_{i 1}\right)=0\right)$ y varianza constante $\left(E\left(\varepsilon_{i 0}^{2}\right)=E\left(\varepsilon_{i 1}^{2}\right)=\sigma^{2}\right)$.

Luego, el individuo elije la opción " 1 " si la utilidad de esa opción supera a la otra. Es decir:

$$
Y_{i}=\left\{\begin{array}{l}
1, \Leftrightarrow \mu_{i 1}>\mu_{i 0} \\
0, \Leftrightarrow \mu_{i 0}>\mu_{i 1}
\end{array}\right.
$$

Entonces, en términos probabilísticos:

$$
\begin{aligned}
& \operatorname{Prob}\left(Y_{i}=1\right)=\operatorname{Prob}\left(i_{i 1}>i_{i 0}\right) \\
& \operatorname{Prob}\left(Y_{i}=1\right)=\operatorname{Prob}\left(X_{i 1} \beta+\varepsilon_{i 1}>X_{i 0} \beta+\varepsilon_{i 0}\right) \\
& \operatorname{Prob}\left(Y_{i}=1\right)=\operatorname{Prob}\left(\varepsilon_{i 1}-\varepsilon_{i 0}>-X_{i 1} \beta+X_{i 0} \beta\right) \\
& \operatorname{Prob}\left(Y_{i}=1\right)=\operatorname{Prob}\left(\Delta \varepsilon>-\left(X_{i 1}+X_{i 0}\right) \beta\right) \\
& \operatorname{Prob}\left(Y_{i}=1\right)=\operatorname{Prob}(\Delta \varepsilon>-X \beta)
\end{aligned}
$$


Esquematizando, se tiene:

$\mu^{1}$ (Visita cueva de las lechuzas) $>\mu^{0}$

(No visita cueva de las lechuzas) (4)

Para medir el cambio en el bienestar de un individuo, derivado de que visita la cueva de las lechuzas, se tendría que indagar sobre su disponibilidad de pago por acceder a dicha mejora. El pago implicaría una reducción en el ingreso disponible del individuo. Actuando de manera racional, él tendría que decidir si compra el bien ambiental - disfrute del ecosistema de la cueva de las lechuzas - y a qué precio, o si no lo compra. Si compra, acepta que tendrá que abstenerse de adquirir otros bienes porque su ingreso es limitado. (Uribe Botero, Mendieta López, \& otros, 2003, pág. 85)

Suponiendo que el bien que se ofrece se representa por la letra $q$ y el ingreso disponible del individuo se representa por la letra $y_{d}$. Entonces el individuo sería indiferente entre comprar el bien o no comprarlo; sí y sólo sí:

$$
\mu^{1}\left(y_{d}-P a g o, q^{1}\right)>\mu^{0}\left(y_{d}, q^{0}\right)
$$

Donde:

$q^{1}=$ Es la situación de goce de la cueva de las lechuzas.

$q^{0}=$ Es la situación sin goce de la cueva de las lechuzas.

El cambio en utilidad, en términos monetarios, podría medirse a partir de la disponibilidad a pagar que tiene el individuo por acceder a los beneficios del bien ambiental ofrecido.

Una vez explicada la racionalidad económica del método de valorización contingente, lo siguiente es tratar de especificar el anterior planteamiento en términos de una función que pueda ser estimable mediante estudios empíricos. Para esto es necesario proponer una forma funcional para la utilidad del individuo que se puede expresar como:

$$
\mu\left(y_{d}, q\right)=V\left(y_{d}, q\right)+\varepsilon
$$

Donde, $V\left(y_{d}, q\right)$ representa la función de utilidad indirecta. Es decir, la función que representa la máxima utilidad que puede alcanzar el individuo dados unos precios y un ingreso disponible. Este concepto es importante en economía del bienestar porque de esta función parte la medición del bienestar ya sea por un cambio en precios o como en este caso, por un cambio en la calidad o cantidad de un bien.

El término $\mathcal{E}$, representa aquella parte de la utilidad que no puede ser explicada por las variables incluidas en el modelo.

Siguiendo este planteamiento, las funciones de utilidad bajo el estado inicial-sin goce de la cueva de las lechuzas- y bajo el estado final-con goce de la cueva de las lechuzas-estarían descritas en las ecuaciones (7) y (8), respectivamente:

$$
\begin{aligned}
& \mu^{0}\left(y_{d}, q^{0}\right)=V^{0}\left(y_{d}, q^{0}\right)+\varepsilon_{0} \\
& \mu^{1}\left(y_{d}, q^{1}\right)=V^{1}\left(y_{d}, q^{1}\right)+\varepsilon_{1}
\end{aligned}
$$

De acuerdo con Haneman (1984), se asume que el valor esperado del error es cero; así el cambio en la utilidad se mide como la diferencia entre la utilidad indirecta en la situación final - con goce del Ecoturismo - menos la utilidad indirecta en la situación inicial - sin goce. Es decir:

$$
\Delta V=V^{1}\left(y_{d}-D A P, q^{1}\right)-V^{0}\left(y_{d}, q^{0}\right)
$$

Ahora sólo resta asignar una forma funcional operable en términos empíricos para la función de utilidad indirecta y luego presentar el modelo econométrico para la estimación. Haneman (1984) y Cameron (1988) proponen una forma funcional lineal que depende del ingreso $\left(y_{d}\right)$ :

$$
V=\alpha+\beta y_{d}
$$

Entonces, la utilidad indirecta inicial y final se representan, para el estado inicial y estado final mediante las ecuaciones (11) y (12), respectivamente:

$$
\begin{aligned}
& V^{0}=\alpha_{0}+\beta y_{d} \\
& V^{1}=\alpha_{1}+\beta\left(y_{d}-D A P\right)
\end{aligned}
$$

El cambio en utilidad se puede ahora expresar como:

$$
\Delta V=\left[\alpha_{1}+\beta\left(y_{d}-D A P\right)\right]-\left(\alpha_{0}+\beta y_{d}\right)
$$

Dado que tanto como representan intercepto, ellos pueden ser adicionados; se tiene que:

$$
\alpha=\alpha_{1}-\alpha_{0}
$$


Por consiguiente,

$$
\Delta V=\alpha+\beta D A P
$$

Al final, sí con el pago que hace el individuo éste queda indiferente entre el nivel de utilidad inicial y el final, es decir si $\Delta V=0$, entonces la disponibilidad a pagar por el bien ofrecido se puede despejar de la siguiente manera (Uribe Botero, Mendieta López, \& otros, 2003, pág. 86):

$$
\begin{aligned}
& 0=\alpha-\beta D A P \\
& \text { Entonces: } \\
& D A P=\alpha / \beta
\end{aligned}
$$

La anterior medida de bienestar es conocida con el nombre de "disponibilidad a pagar media (DAP)", representa la cantidad de dinero que el individuo representativo está dispuesto a pagar por el bien ofrecido.

En los modelos empíricos, la forma funcional representada puede ser estimada junto con variables socioeconómicas $(\mathrm{z})$. Una formulación típica de este tipo de modelos es:

$$
\operatorname{Pr} o b(\mathrm{SI}=1)=\beta_{0}-\beta_{1} D A P+\sum_{i=1}^{N} \beta_{i} z_{i}(18)
$$

La fórmula para estimar la disponibilidad a pagar media para estos modelos es (Uribe Botero, Mendieta López, \& otros, 2003, pág. 87):

$$
D A P=\frac{\beta_{0}+\sum_{i=1}^{N} \beta_{i} z_{i}}{\beta_{1}}
$$

\section{METODOLOGÍA}

La investigación se desarrolló sobre la base de una encuesta, aplicada a una muestra aleatoria de 200 personas, tomada de una población indefinida (visitantes). Los datos recogidos fueron procesados y usados como insumos para la estimación del modelo econométrico de elección discreta tipo PROBIT, utilizado para contrastar la empírica de la hipótesis de investigación.

\section{RESULTADOS}

En los últimos años las regiones del Perú han ido convirtiéndose en lugares de destino para el turista nacional y extranjero esto se debe a la biodiversidad incomparable de nuestro país. Los resultados obte- nidos muestran el comportamiento que cada turista tiene y su preferencia por el servicio ambiental va desde investigación hasta fines recreacionales.

\section{Tabla No 01}

Sexo, nivel educativo, tipo de empleo y carga familiar de los Turistas afluentes a la Cueva de las Lechuzas

\begin{tabular}{|l|c|c|}
\hline \multicolumn{1}{|c|}{$\begin{array}{c}\text { Aspecto } \\
\text { Socioeconómico }\end{array}$} & Turista & $\begin{array}{c}\text { \% de } \\
\text { Turistas }\end{array}$ \\
\hline \multicolumn{3}{|c|}{ Sexo } \\
\hline Femenino & 92 & 46 \\
\hline Masculino & 108 & 54 \\
\hline \multicolumn{3}{|c|}{ Nivel educativo } \\
\hline Escolar & 103 & 52 \\
\hline Superior & 97 & 49 \\
\hline \multicolumn{3}{|c|}{ Tipo de empleo } \\
\hline Independiente & 108 & 54 \\
\hline Dependiente & 64 & 32 \\
\hline Estudiante & 28 & 14 \\
\hline \multicolumn{3}{|c|}{ Carga familiar } \\
\hline Menor a 3 & 2 & 1 \\
\hline Entre 3 a 6 & 116 & 58 \\
\hline De 6 a más & 82 & 41 \\
\hline
\end{tabular}

Fuente: Encuesta realizada en el 2016.

Los turistas afluentes a la Cueva de las Lechuzas se caracterizan socioeconómicamente, por aquellos que están dispuestos a pagar un poco más son el $50 \%$ del sexo masculino, así como el $52 \%$ poseen un nivel educativo escolar los que desempeñan un tipo de empleo independiente el 54\% y el 14\% se encuentra hasta el día de hoy estudiando en la universidad o instituto superior. Los turistas presentan una carga familiar promedio de 6 personas, así como esta cantidad el 50\% de ellos es menor o igual, pero con frecuencia la carga familiar es de 5 personas.

El aspecto socioeconómico del turista juega un rol muy importante, ante la decisión de disponer de una cantidad de dinero para contribuir económicamente en el cuidado de la preservación del atractivo turístico de la Cueva de las Lechuzas, por lo que la valoración económica hecha por cada turista tiene implicancia en el ordenamiento racional o lógico de los diferentes bienes y servicios los que pueda renunciar o complementar como parte de su canasta familiar con el fin de obtener un nivel de bienestar económico. 
Tabla No 02:

Edad, ingresos y gastos de los Turistas afluentes a la Cueva de las Lechuzas

\begin{tabular}{|l|c|c|}
\hline $\begin{array}{c}\text { Aspecto } \\
\text { Socioeconómico }\end{array}$ & Turista & $\begin{array}{c}\text { \% de } \\
\text { Turistas }\end{array}$ \\
\hline \multicolumn{2}{|c|}{ Edad (Años cumplidos) } \\
\hline Menor a 28 & 56 & 28 \\
\hline Entre 28 a 39 & 72 & 36 \\
\hline Entre 40 a 49 & 38 & 19 \\
\hline Mayor a 49 Ingresos (En soles) \\
\hline \multicolumn{3}{|c|}{34} \\
\hline Menor a 850 & 34 & 17 \\
\hline Entre 850 a 1500 & 123 & 62 \\
\hline Entre 1500 a 2500 & 36 & 18 \\
\hline Entre 2500 a 3500 & 7 & 3 \\
\hline \multicolumn{3}{|c|}{ Gasto (En soles) } \\
\hline Menor a 330 & 178 & 89 \\
\hline Entre 330 a 600 & 19 & 9 \\
\hline Mayor a 600 & 3 & 2 \\
\hline
\end{tabular}

Fuente: Encuesta realizada en el 2016.

Los turistas que visitan la Cueva de las Lechuzas tienen una edad promedio de 36 años, pero el $50 \%$ de ellos fluctúan entre los 17 a 35 años con frecuencia visitan con edad de 32 años. Sus ingresos fluctúan entre el sueldo mínimo y los 3500 soles, lo que representa el ingreso promedio de 1260 soles mensuales con una frecuencia de 1179 soles, vale indicar que el 50\% de estos turistas afirman tener ingresos a lo mucho de 1199 soles. El gasto promedio por persona durante su permanencia en el lugar es de 196 soles.

\section{Tabla No 03:}

Características de elección de los servicios ambientales por parte de los Turistas afluentes a la

Cueva de las Lechuzas

\begin{tabular}{|l|c|c|}
\hline \multicolumn{1}{|c|}{$\begin{array}{c}\text { Características } \\
\text { de Elección }\end{array}$} & Turista & $\begin{array}{c}\text { \% de } \\
\text { Turistas }\end{array}$ \\
\hline \multicolumn{3}{|c|}{ Visita } \\
\hline Por invitación & 152 & 76 \\
\hline Por voluntad propia & 48 & 24 \\
\hline \multicolumn{3}{|c|}{ Motivo } \\
\hline Recreación & 168 & 84 \\
\hline Investigación & 32 & 16 \\
\hline \multicolumn{3}{|c|}{ Destino } \\
\hline $\begin{array}{l}\text { Cueva de las Lechu- } \\
\text { zas }\end{array}$ & 94 & 47 \\
\hline $\begin{array}{l}\text { Varios lugares turís- } \\
\text { ticos }\end{array}$ & 106 & 53 \\
\hline
\end{tabular}

Fuente: Encuesta realizada en el 2016.
Los turistas eligen un lugar turístico de acuerdo a la iniciativa de visita, como por el motivo y el destino. Es decir, el 76\% visita Cueva de las Lechuzas porque algún familiar o amigo le ha invitado al lugar, la gran mayoría de ellos lo hace por recreación así se corrobora que el $84 \%$ y que un poco menos del $50 \%$ afirma visitar exclusivamente este atractivo turístico.

\section{Figura No 01}

Disponibilidad a pagar por los turistas afluentes a la cueva de las Lechuzas

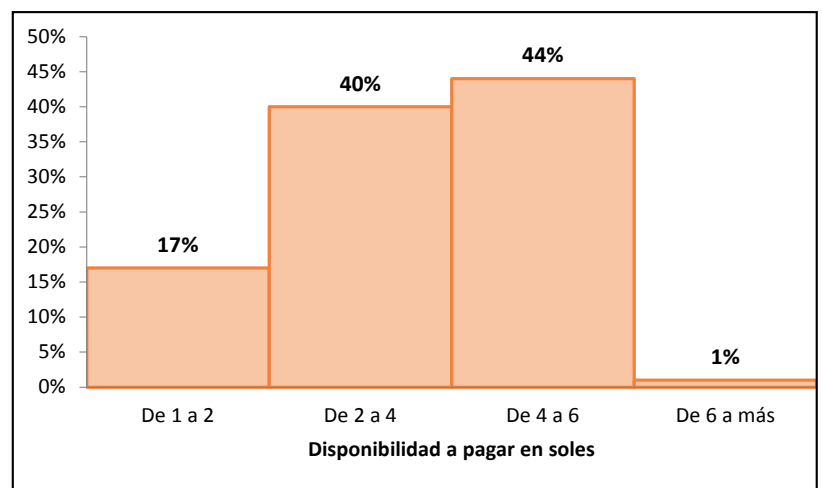

Fuente: Encuesta realizada en el 2016.

Los turistas coinciden que la DAP por disfrutar así como por conservar el ecosistema de la Cueva de las Lechuzas es de 4 soles en promedio; así como con frecuencia afirma este monto y el $50 \%$ valora entre $S / .1$ a 4 .

La Tabla $\mathrm{N}^{\circ} 04$ nos muestra tres tipos de modelos

\section{Tabla No 04:}

Portafolio de modelos de probabilidad de respuesta dicotómica de la DAP por parte de los Turistas afluentes a la Cueva de las Lechuzas

\begin{tabular}{|c|c|c|c|}
\hline \multirow{2}{*}{$\begin{array}{c}\text { Variable } \\
\text { independiente }\end{array}$} & Probit & Logit & Gompit \\
\hline & \multicolumn{3}{|c|}{ Pruebas de bondad de ajuste } \\
\hline $\begin{array}{l}\text { Log. del índice de } \\
\text { máxima verosimi- } \\
\text { litud }\end{array}$ & $-46,725$ & $-47,575$ & $-47,429$ \\
\hline $\begin{array}{l}\text { Chi-cuadrado } \\
\text { empírico }\end{array}$ & $178.666^{* *}$ & $176.966^{* *}$ & $176.966^{* *}$ \\
\hline $\begin{array}{l}\text { Criterio de informa- } \\
\text { ción de Akaike } \\
\end{array}$ & 0.597 & 0.606 & 0.604 \\
\hline $\begin{array}{l}\text { Criterio de informa- } \\
\text { ción de Schwarz } \\
\end{array}$ & 0.812 & 0.820 & 0.819 \\
\hline $\begin{array}{l}\text { Criterio de infor- } \\
\text { mación de Han- } \\
\text { nan-Quinn }\end{array}$ & 0.684 & 0.693 & 0.691 \\
\hline R2 de McFadden & 0.657 & 0.650 & 0.651 \\
\hline
\end{tabular}

Vol. 24(46) 2016 | QVIPURAMAYOC / 167 
probabilísticos de respuesta dicotómica de la DAP, de los cuales se debe de elegir uno de ellos que mejor ajuste presenta en los datos recogidos en base a las encuestas hecha a los turistas afluentes a la Cueva de las Lechuzas, por lo que el modelo Probit presenta todas estas exigencias, respecto a las pruebas de bondad de ajuste el logaritmo del índice de máxima verosimilitud es mayor para el modelo Probit, para la explicación para este tipo de modelo, lo propio ocurre para la prueba de relevancia global medido por la distribución chi-cuadrado que el resultado es estadísticamente muy significativo $(\mathrm{p}<0.01),(\mathrm{p}<0.05)$; respecto, a los criterios de información de Akaike, Schwarz y Hannan-Quinn sus resultados nos indican que el Probit es de su mejor elección que responden a la mejor estimación para este tipo de modelo y según el coeficiente de determinación de McFadden desde luego el PROBIT es la que mejor que explica la variabilidad de todas las variables independientes sobre la variabilidad en la probabilidad de la DAP.

Todas las variables independientes con ex-

\section{Tabla No 05}

Modelo PROBIT de respuesta dicotómica de la DAP por parte los Turista afluentes a la Cueva de las

Lechuzas

\begin{tabular}{|l|l|l|}
\hline \multicolumn{1}{|c|}{$\begin{array}{c}\text { Variable } \\
\text { independiente }\end{array}$} & \multicolumn{1}{|c|}{$\beta \mathrm{i}$} & \multicolumn{1}{c|}{$\begin{array}{c}\text { Desviación } \\
\text { estándar }\end{array}$} \\
\hline Intercepto & $-8.272^{* *}$ & 1,814 \\
\hline Edad & $-0.07^{* *}$ & 0.018 \\
\hline Sexo & $0.808^{*}$ & 0.328 \\
\hline Educación & $0.722^{*}$ & 0.722 \\
\hline Empleo & $-0.496^{*}$ & -0.496 \\
\hline Ingreso & $2.788^{* *}$ & 2,788 \\
\hline Gasto & $-0.004^{*}$ & -0.004 \\
\hline Visita & $1.358^{* *}$ & 1,358 \\
\hline Motivo & 0.325 & 0.325 \\
\hline Destino & $1.337^{* *}$ & 1,337 \\
\hline Carga familiar & $-0.251^{*}$ & -0.251 \\
\hline *p $<0.05$ & & \\
**p $<0.01$ & & \\
\hline
\end{tabular}

cepción de Visita los resultados nos muestra una relación explicativa de causa-efecto con la DAP estadísticamente muy significativa $(\mathrm{p}>0.01), \mathrm{p}(>0.05)$. Las pruebas de redundancia y omisión de variables para este modelo se concluye para cada una de las variables explicativas, rechazando la hipótesis nula de la no existencia de redundante que es la variable y aceptando la hipótesis nula de que no existe omisión de variables, tampoco existe estadísticamente significativa $(\mathrm{p}<0.01),(\mathrm{p}<0.05)$ la presencia de heterocedasticidad.

Tomando en cuenta la elasticidad como análisis se encontraron los siguientes resultados: si la edad del turista aumentase en un año más, entonces, la probabilidad de que la disponibilidad de pago aumentase es del 2.04\%, mientras que los Turistas del sexo masculino están dispuestos a pagar más que los del sexo femenino, ya que, la probabilidad es mayor en un $23.04 \%$, comparando a un turista con nivel educativo superior con otro que tiene nivel educativo escolar la probabilidad de la disponibilidad a pagar es mayor en $29.49 \%$. El turista que tiene un empleo de tipo independiente, su probabilidad de la disponibilidad a pagar es menor a $27.35 \%$ respecto a otro que es dependiente. Si el ingreso de los turistas aumentase por cada 1000 soles tendría más disponibilidad, lo cual se traduce aumentando la probabilidad en la disponibilidad de pago en $96.19 \%$ pero, si sus gastos aumentasen los turistas desde el lugar de origen a la cueva esto se vería afectado en una menor probabilidad de pago en $0.13 \%$, lo propio ocurre con la carga familiar si aumentase esta entonces la probabilidad de pago disminuye en $7.88 \%$. Los turistas que visitan por recreación están dispuestos a pagar más en $7.18 \%$ respecto a los que visitan por investigación, más aún se incrementa esta disponibilidad de pago en $41.48 \%$ si la decisión es solo visitar la Cueva de las Lechuzas frente a aquellos que visitarían varios lugares a la misma vez. Finalmente, el modelo PROBIT nos sugiere que la DAP por los Turistas nacionales y extranjeros es de $S / .3$ y 5 respectivamente.

\section{CONCLUSIONES}

1. Los principales factores que determinan el valor económico del ecoturismo en la zona de la Cueva de las Lechuzas, corresponden a las variables referidas a los aspectos socioeconómicos tales como: edad, sexo, carga familiar, nivel educativo, tipo de empleo, ingreso promedio mensual y gasto promedio diario de permanencia en el lugar.

2. Con relación a las características de la elección de los servicios ambientales se encontró que el principal

168/ QVIPURAMAYOC | Vol. 24(46) 2016 
motivo de la visita a este importante recurso turístico corresponde no a iniciativa propia, sino más bien a la invitación de un amigo y/o familiar que antes ya visitó la zona. Respecto, al destino de la visita, la mayoría de los encuestados coincidieron en responder que la visita a la Cueva de las Lechuzas no constituye el único destino, sino más bien uno de otros lugares existentes en la zona. Y, por último en cuanto en lo que se refiere al tipo de turismo, el grueso de los visitantes señala realizar la práctica de un turismo recreativo, seguido por aquellos que realizan turismo de carácter científico.

3. La explotación de los recursos ambientales del ecoturismo de la cueva de las lechuzas, con mayor frecuencia por recreación y por investigación, es de poca importancia.

4. La disponibilidad a pagar y aceptar por el uso de los servicios ambientales y su mejora de ello es de 3 soles y 5 soles por turista nacional y extranjero respectivamente.

5. Los proyectos productivos y de infraestructura deben de estar estrechamente relacionados con el medio ambiente en cuanto a la preservación y conservación del equilibrio ambiental en la zona turística

\section{REFERENCIAS BIBLIOGRÁFICAS}

1. Azqueta, D. (1994). Valoración económica de la calidad ambiental. Madrid: McGraw-Hill.

2. Cameron, T. A. (1988). A New Paradigm for Valuing Non-Markets Goods Using Referendum Data: Maximun Likelihood Estimation by Censored Logistic Regression. Journal of Environmental Economics and Management, 15(3), 335-379.

3. Colin Cameron, A., \& Trivedin, P. K. (2005). Microeconometrics methods and applications. Cambridge: Cambridge University.

4. Galarza Contreras, E. (2004). La economía de los recursos naturales. Lima: CIUP.

5. Greene, W. H. (1999). Análisis Econométrico (Tercera ed.). Madrid: Pearson Educación.

6. Haneman, W. M. (1984). Welfare Evaluations in Contingent Valuation Experiments with Discrete Responses. Amer J., of Agr. Econ., 332-341.

7. Uribe Botero, E., Mendieta López, J. C., \& otros. (2003). Introducción a la Valoración Ambiental y Estudios de Caso. Bogotá: Ediciones Uniandes. 